\title{
Применение нейронных сетей для нахождения дискретного спектра прямой задачи Захарова-Шабата
}

\author{
Е.В. Седов ${ }^{1, *}$, И.С. Чеховской ${ }^{1,2}$ \\ ${ }^{1}$ Новосибирский государственный университет, г. Новосибирск \\ ${ }^{2}$ Федеральный исследовательский цеентр информациооных и вычислительных технологий, \\ 2. Новосибирск \\ *E-mail: e.sedov@g.nsu.ru
}

DOI: 10.31868/RFL2020.199-200

Оптические телекоммуникации в настоящее время активно развиваются, однако перманентное увеличение объемов передаваемого трафика в ближайшем будущем превысит потенциальные возможности линий связи, основанных на текущих разработках. В связи с этим, активно исследуются новые перспективные способы увеличения пропускной способности линий связи. В частности, недавно было предложено использовать для передачи данных нелинейное преобразование Фурье (NFT) [1]. Прямое NFT для нелинейного уравнения Шредингера (НУШ) состоит в сопоставлении оптическому сигналу его нелинейного спектра (решении прямой задачи Захарова-Шабата), состоящего из дискретной и непрерывной части. Набор дискретных собственных значений (д. с. з.) соответствует солитонной части сигнала. Данное представление удобно тем, что оно тривиальным образом эволюционирует при распространении сигнала. При любом значении эволюционной переменной с помощью обратного NFT сигнал может быть полностью восстановлен. Передача данных с помощью NFT позволит естественным образом учесть влияние нелинейных эффектов при распространении оптического сигнала по волокну.

Основная сложность на пути широкого распространения NFT заключается в нехватке быстрых и точных численных методов для его нахождения. На данный момент предложено большое количество методов для определения нелинейного спектра и был достигнут существенный прогресс в сокращении сложности алгоритмов и увеличении их точности. Однако при применении к сложным сигналам остаются проблемы с устойчивостью вычислительных алгоритмов. Также затруднительным остается вычисление в реальном времени NFT для сигналов сложной формы, что ограничивает возможности реализации NFT на аппаратном уровне в современных линиях связи.

Многообещающим направлением в данном случае выглядит применение машинного обучения, в частности нейронных сетей. В последние годы произошел большой скачок в развитии методов машинного обучения для решения алгоритмически сложных задач, таких как, например, распознавание и классификация изображений. Основные этапы при этом - обучение модели на основе набора некоторых данных, и применение модели для получения предсказания. Первый этап может занимать длительный отрезок времени. Однако применение обученной модели обычно осуществляется намного быстрее, что позволяет реализовывать системы на основе методов машинного обучения на различных устройствах с низкой производительностью.

В последние годы машинное обучение также было предложено использовать в системах передачи данных на основе NFT на этапе постобработки [2]. В нашей работе предлагается реализовать более кардинальный подход и вычислять само NFT с помощью нейронных сетей. 

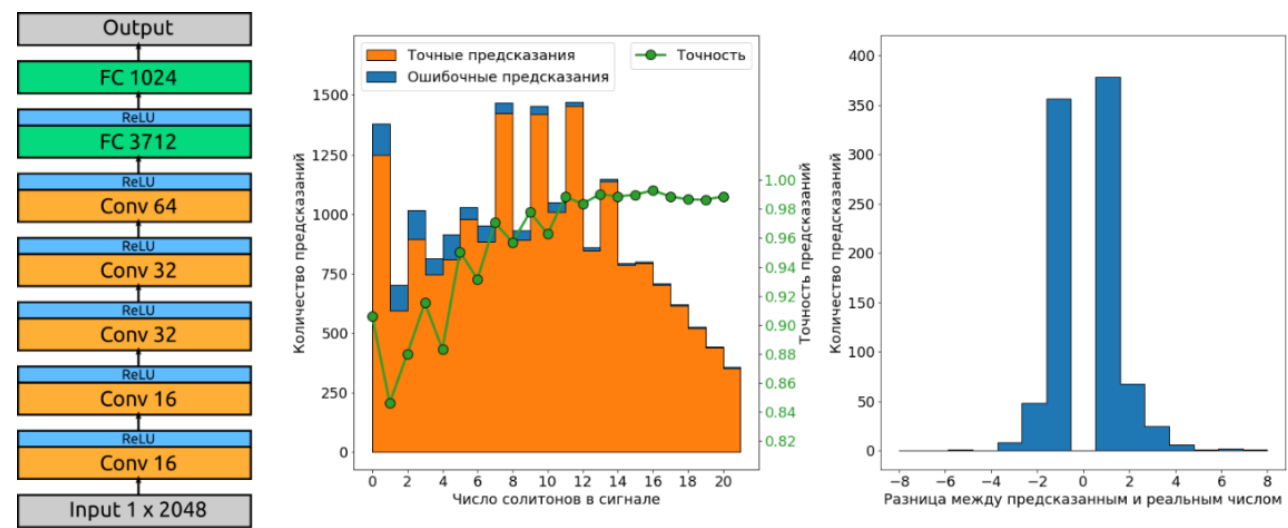

Рис. 1. Архитектура нейронной сети и точность ее предсказания.

В рамках данной работы нейронная сеть использовалась для предсказания числа д.с.3. в нелинейном спектре телекоммуникационных сигналов. Дискретные с. з. отражают внутреннюю структуру сигнала. Знание о внутренней структуре сигнала дает возможность для изучения его свойств и характера распространения в оптическом волокне.

В качестве изучаемого сигнала был выбран WDM формат, который широко используется в оптической коммуникации. Сигнал формировался из случайного набора данных, закодированного по одному из форматов модуляции: QPSK, 16QAM, 64-QAM, 1024-QAM. За основу архитектуры сети был выбран упрощенный вариант сети VGG-16, которая используется в задачах распознавания изображений. На входе сеть принимает комплексный сигнал, состоящий из 1024 точек. Этот сигнал преобразуется в вектор с 2048 элементами, в котором последовательно расположены действительные и мнимые части каждой точки начального комплексного сигнала. Далее сигнал обрабатывается несколькими сверточными слоями с функциями активации и полносвязными слоями. На выходе сети выдается количество солитонов в сигнале. Количество тренируемых параметров в сети составило 3834145 .

Всего в тренировочном наборе было 174847 сгенерированных сигналов, в которых содержатся от 0 до 20 солитонов включительно. Для каждого сигнала это число заранее посчитано другими методами. Точность сети определялась на валидационном наборе из 19427 сигналов. Сеть тренировалась в течении 300 эпох, финальная точность предсказаний на валидации составила 95.39\%. При этом максимальная “ошибка" работы сети - разница между реальным числом солитонов в сигнале и предсказанным - составила 8. Большинство ошибочных результатов находятся в диапазоне [-2;2]. Лучше всего сеть работает для сигналов, где количество солитонов было больше 10. Для таких случаев точность выше 98\%. Хуже всего определялись сигналы, где только один солитон - для них точность составила $84 \%$. Полученные результаты показывают, что нейронные сети имеют большой потенциал для реализации с их помощью различных этапов NFT.

Исследование выполнено при поддержке фонда Президента РФ для государственной поддержки молодых российских учёных (грант № МК-677.2020.9). Работа Седова Е.В. была поддержана государственным заданием на проведение фундаментальных исследований FSUS-2020-0034.

\section{Литература}

[1] S. K. Turitsyn, J. E. Prilepsky et al, Optica, 4, 307-322 (2017)

[2] O. Kotlyar, M. Pankratova et al, Opt. Lett., 45, 3462 (2020) 